\title{
A prospective study on fungal infection in children with cancer
}

\author{
H. A. El-MAhallawy, I. Attia, N. H. Ali-El-Din, A. E. SAlem, S. ABO-El-NAGA \\ Chest Department, National Cancer Institute, Cairo University, Cairo, Egypt
}

\begin{abstract}
A prospective study was conducted in 1999 at the National Cancer Institute, Cairo University, to estimate the incidence, morbidity and mortality of fungal infections along with the evaluation of risk factors influencing outcome of infections among paediatric cancer patients. Of 1917 infectious episodes, the fungal infection rate as documented both clinically and microbiologically was $3.7 \%$ (70 cases). Fungal pathogens isolated were yeasts in 55 patients $(\mathbf{7 8 . 6} \%)$ and moulds in 15 patients $(21.11 \%)$. Among yeasts, Candida parapsilosis was the commonest, followed by $C$. tropicalis. Pneumonia was the most common fungal infection $(n=25,35.7 \%)$, followed by fungaemia $(n=18,25.7 \%)$. The overall mortality rate was $40 \%(n=28)$, with an infection-related mortality of 28.5\% $(n=20)$. Risk factors that accompanied mortality were relapsing or recurrent disease, profound neutropenia, ADE (Ara-C, daunorubocin and etoposide) protocol of chemotherapy, $C$. tropicalis isolated and fungaemia as a site of infection. Early use of empirical antifungal therapy (day 4) was not associated with a better outcome. In the light of the poor outcome of patients with fungaemia and fungal pneumonia, every effort should be made to prevent these infections in paediatric cancer patients.
\end{abstract}

\section{Introduction}

Advances in medical therapy have greatly improved the prognosis for patients with cancer. While progress has been made in eradicating malignant disease, a growing concern for patients who receive cytotoxic chemotherapy is the development of fungal infections. At some centres, fungal infections are the leading cause of death in patients who are receiving chemotherapy for leukaemia [1]. Various factors account for the increased frequency of fungal infections in cancer patients. More intensive regimens are being used for the treatment of cancer patients and these dose-intensive regimens are associated with more profound neutropenia and mucosal barrier damage [2]. Improved control of infections by the widespread use of antibiotics has reduced mortality from gram-negative bacteraemia but has increased the likelihood of fungal super-infection. Moreover, the placement of indwelling central venous catheters (CVCs) has become a routine part of supportive care during therapy for cancer [3]. Thus, cancer patients are vulnerable to fungal infections.

Received 9 April 2001; revised version received 2 Jan. 2002; accepted 31 Jan. 2002.

Corresponding author: Dr H. A. El-Mahallawy (e-mail: hadir38@hotmail.com or zeneldin@ie-eg.com).
Candida spp. continue to be the most common fungal pathogens in patients with cancer. They account for $c$. $75 \%$ of fungal infections, most of which have been attributed previously to C. albicans [4]. Recently, other Candida species such as C. tropicalis, C. parapsilosis and $C$. lusitaniae have also been implicated in fungal infections in immunocompromised individuals [5]. This study was performed in the paediatric oncology department at the National Cancer Institute, Cairo University, to evaluate the incidence of fungal infections, to identify the type of causative fungi and to identify the risk factors that influence the outcome of such infections.

\section{Patients and methods}

This prospective study was performed in the paediatric wards of the National Cancer Institute, Cairo University, a university-based tertiary care centre, during the period Jan.-Dec. 1999.

\section{Patient population}

Patients were included only if they were symptomatic. Febrile patients with or without neutropenia, and patients with localised sites of infection were the 
subjects of this study. A total of 1917 infectious episodes was analysed. Fungal pneumonia was diagnosed clinically, by imaging, and microbiologically documented.

The patients with positive fungal growth were evaluated clinically for preceding chemotherapy including administration of corticosteroids, the extent of neutropenia, duration of hospitalisation and extent of disease. Nosocomial infections were reported in the patients if they were hospitalised for 4 days before the occurrence of fever. Response to antimicrobial therapy was evaluated at days 4, 7 and 14 and weekly thereafter for up to 30 days if the patient was experiencing the same episode of infection.

\section{Methods}

For blood cultures, blood samples were collected from two peripheral veins. If the cannula, portacath or CVC was suspected as the source of infection, a blood sample was obtained from the catheter and another from a peripheral vein. Collected blood was injected directly into Bactec $^{\circledR}$ (Becton Dickinson, USA) culture vials. Vials were incubated in the Bactec $^{\circledR} 9050$ incubator after collection [6]. Bronchial lavage samples were obtained by tracheal aspiration or bronchoscopy and broncho-alveolar lavage (BAL) whenever invasive procedures were not risky due to thrombocytopenia, in addition to collection of sputum samples. Other samples obtained were cerebrospinal fluid (CSF), urine and stool.

Positive fungal growth necessitated the processing of another sample if possible from the same patient to exclude contamination. Yeasts were identified by standard and biochemical criteria and by the Fungifast Twin kits (International Microbio, Stago Group, France) [7]. Mixed growth of gram-positive and gram-negative organisms was recorded.

\section{Statistical analysis}

$\chi^{2}$ and Fisher's exact tests were performed with SPSS software, version 9.0. Logarithmic transformation was done for the absolute neutrophil count (ANC) and comparisons were performed on $\log$ values. $\chi^{2}$ results were two-sided and $\mathrm{p}$ values $<0.05$ were considered significant.

\section{Results}

During the 1-year period, 1917 infectious episodes were cared for in the paediatric department of the National Cancer Institute, Cairo University. Fungal infections were diagnosed clinically and by culture in 70 cases. Fungi were isolated from the following specimens: respiratory (30 cases), blood and intravascular (i.v.) device cultures (30), CSF (5), urine (3) and stool (2). The fungi isolated were yeasts in 55 cases and moulds in 15. Pure fungal growth was observed in 29 patients, whereas mixed bacterial and fungal growth was encountered in 41 cases. The Candida species isolated are listed in Table 1. Chromagar medium was able to identify C. albicans and C. tropicalis. Aspergillus spp. were isolated from 13 cases, Histoplasma capsulatum in one case and Fusarium sp. in one case.

\section{Demographic characteristics of patients}

The mean age of the patients was 9.3 SD 4.4 years. The male to female ratio was $1.3: 1$. The most common underlying immunodeficiency was leukaemia; 40 patients were diagnosed with acute leukaemia, acute lymphoblastic leukaemia (ALL) in 22 and acute nonlymphoblastic (myeloid) leukaemia (ANLL) in 18. The underlying disease in the remaining patients was lymphoma in 15 and solid tumours in another 15 . The state of disease at time of occurrence of the febrile episode was relapsing or recurrent disease in 36 cases, complete remission in 19 cases and during the induction phase of chemotherapy in 15 cases.

\section{Epidemiology}

Fifty-three patients $(75.7 \%)$ had neutropenia: $<1000$ neutrophils $/ \mu 1$, ranging between 500 and $100 / \mu 1$ in 15 cases and $<100 / \mu 1$ in 34 cases. Sixty-six patients had received chemotherapy; 23 of them had received corticosteroids as well in the month before the diagnosis of fungal infection. Nosocomial infections were recorded in 48 patients $(68.5 \%)$.

\section{Clinical presentation}

The initial clinical symptom or abnormality developed within a mean of 8.9 SD 6.2 days after receiving chemotherapy. Fever was the main presenting symptom in 62 patients with a mean of $38.9 \mathrm{SD} 0.9^{\circ} \mathrm{C}$. Peripheral blood cultures were positive for fungal cultures in 18 cases, whereas i.v. device blood cultures were positive for fungi in 12 cases with a negative peripheral vein

Table 1. Types of fungal pathogens isolated

\begin{tabular}{lc}
\hline Fungal isolates & Number $(\%)$ \\
\hline Yeasts & $55(78.6)$ \\
C. parapsilosis & $14(24.4)$ \\
C. tropicalis & $13(23.6)$ \\
Cr. neoformans & $7(12.7)$ \\
C. albicans & $5(9.0)$ \\
C. lusitaniae & $4(7.2)$ \\
C. guilliermondii & $3(5.4)$ \\
C. glabrata & $3(5.4)$ \\
C. krusei & $3(5.4)$ \\
C. kefyr & $1(1.8)$ \\
Unidentified & $2(3.6)$ \\
Moulds & $15(21.4)$ \\
Aspergillus spp. & $13(86.7)$ \\
Fusarium sp. & $1(6.7)$ \\
H. capsulatum & $1(6.7)$ \\
\hline
\end{tabular}


culture. Infected i.v. devices were i.v. cannula site in six cases, portacath and CVC in three cases each. Fungi isolated were mostly $C$. parapsilosis and Cryptococcus neoformans. Cr. neoformans was isolated from five patients during a 1-month period and cases were hospital-acquired. Patients with fungaemia were all febrile with temperatures of $\geqslant 39^{\circ} \mathrm{C}$. C. tropicalis and Aspergillus spp. were the commonest pathogens isolated from blood cultures; they were found in seven and eight cultures, respectively.

\section{Respiratory tract infections}

Fifteen patients suffered severe mucositis, which usually preceded either oesophagitis or chest infection. Oesophagitis was diagnosed in eight cases and was accompanied by a normal chest X-ray in six of them. Fungal pathogens isolated from respiratory tract infections were Aspergillus spp. (six cases), C. parapsilosis (seven), C. tropicalis (four), C. albicans (four), C. lusitaniae (two), C. kefyr (one) and H. capsulatum (one). It is worth mentioning that one patient had chronic disseminated candidosis manifested by right pulmonary emphysematous bulla, hepatic candidosis and cachexia. Only one patient with a respiratory tract infection presented with sinusitis and this was complicated by a perforated nasal septum.

\section{Central nervous system infection}

Fungi were isolated from the CSF of five patients. Fever, headache and convulsions were the presenting features.

\section{Urinary tract and gastrointestinal tract infections}

Three patients presented with dysuria and haematuria after catheterisation. Catheter removal, antibiotics but no antifungal drugs were the main control measures used for these patients. Fungi were isolated from stool specimens of two lymphoma patients who were found to have disease infiltrating their intestines by imaging studies.

\section{Empirical antifungal agents}

An empirical antifungal agent was administered to 49 patients: fungizone in 28 cases, fluconazole in 16 and both in 5 cases. Nineteen of the patients received the antifungal agent at day 4 whereas 30 cases received it at day 7. No significant difference was found between starting antifungal therapy on day 4 or day 7 . A poor prognosis was observed in 6 of 17 and 12 of 30 of those who received antifungal therapy at day 4 and day 7 , respectively $(p=0.38)$. All patients with isolated fungi localised to i.v. lines were successfully managed by removal of the line and administration of an antifungal agent.

\section{Outcome of infectious episode}

Fungaemia was accompanied by $72.2 \%$ overall mortality (13 of 18 patients). Clinical and laboratory evidence indicated infection-related mortality in 12 cases, whereas one patient showed evidence of disease progression rather than infection. Twelve of the patients with a respiratory tract infection died, with infectionrelated mortality in eight of them. Unfavourable outcomes in other sites were more related to disease progression than infections.

\section{Risk factors}

Risk factors associated with fungal infections in relation to outcome were as follows.

Although a better outcome of fungal infection was encountered in patients with solid tumours and lymphoma than those with acute leukaemia, as 7 of 30 in the former group versus 19 of 40 of the latter died following infection, the $\mathrm{p}$ value did not reach a significant level.

The state of disease had an impact on outcome, as 19 of 36 patients with a relapsing disease died in contrast to 2 of 19 experiencing complete remission $(\mathrm{p}=0.002)$.

ANC was lower among patients who died $(\mathrm{p}=0.046)$. ANC showed a median of $220(0-20,730 / \mu \mathrm{l})$ in surviving patients and median of $100(0-20,000 / \mu 1)$ in those who died.

Chemotherapy was difficult to compare in all patients due to diversity of protocols. Eight of 11 patients who received ADE (Ara-C, daunorubocin and etoposide) died versus 17 of 52 receiving different protocols $(p<0.0001)$. Patients receiving corticosteroids were more associated with persistent infections or unfavourable outcome in $78.3 \%$ versus $55.6 \%$ of those who were not on steroids, with borderline significance at $\mathrm{p}=0.06$.

Hospital-acquired infection had a negative impact on the outcome of infectious episodes, as 22 of 48 patients with nosocomial infections died in contrast to 5 of 22 of those whose infections were not nosocomially acquired, with a $\mathrm{p}$ value of borderline significance at 0.06 .

As regards site of infection, fungaemia showed the highest related mortality when compared with other sites of infection $(\mathrm{p}<0.0001)$.

C. tropicalis was associated with the highest related mortality compared with other isolates $(p=0.002)$.

Patients with a pure fungal infection showed a better 
response and outcome than those with mixed infections, but the result did not reach a significant level.

\section{Discussion}

Fungal infections are an important cause of morbidity and mortality in patients with acute leukaemia. Candidaemia, once rare, is now a common nosocomial infection because of the intensity of chemotherapy, prolonged neutropenia, administration of broad-spectrum antibiotics and use of CVCs [5]. Fungal infections affected $3.7 \%$ of all paediatric cases treated with chemotherapy at the National Cancer Institute, Cairo University.

Considerable progress has been made in blood culture technologies, allowing earlier detection of the aetiological agents, especially Candida spp. [6]. The findings of the present study confirmed that Candida spp. accounted for the majority of infections, as the fungal pathogen isolated was a yeast in nearly $80 \%$ of the cases. Similarly, it has been reported that Candida spp. account for $75 \%$ of fungal infections in patients with cancer [8]. In the present study, non-albicans Candida species were more predominant than $C$. albicans as a cause of fungal infections. The most frequently isolated species were $C$. parapsilosis and $C$. tropicalis, accounting for $25.5 \%$ and $23.6 \%$, respectively; followed by $\mathrm{Cr}$. neoformans in $12.7 \%$. C. albicans accounted for only $9 \%$ of candidal infections.

These results are different from studies done in cancer centres in various other areas, as C. albicans has long been recognised as the most common cause of disseminated candidosis $[5,9,10]$. Although increasing rates of infection due to non-albicans Candida have been reported, $C$. albicans still causes c. $50 \%$ of cases of bloodstream infections in large series [9]. In an assessment of the frequency and distribution of nonalbicans Candida spp. among patients with cancer, 37 reports that described 1591 cases of systemic candida infections were reviewed. Species other than candida accounted for $46 \%$ of these infections in patients with cancer. C. tropicalis, C. glabrata, C. parapsilosis and C. krusei accounted for $25 \%, 8 \%, 7 \%$ and $4 \%$, respectively [4]. Moreover, C. albicans was isolated in $70 \%$ of 249 candidaemia episodes in patients with solid tumours and 36\% involving those with haematological disease in a surveillance study in cancer patients conducted by the European Organization for Research and Treatment of Cancer [11].

The most frequent risk factors for invasive fungal infections were reported to be neutropenia, previous therapy with multiple antibiotics and recent catheter insertion [12,13]. The findings in the present study suggest that neutropenia, acute leukaemia, relapsing disease, ADE protocol of chemotherapy and nosocomial infection are risk factors for poor outcome of fungal infections. Therapy with corticosteroids was found to be more frequent with persistent fungal infections in this group of patients. Similarly, it has been reported that steroid therapy occurred more frequently in the paediatric subgroup with fungaemia [14].

Of all patients with fungal infections, disseminated bloodstream infections were the most dangerous. Unfortunately, early antifungal therapy at day $4 \mathrm{did}$ not affect the unfavourable outcome of fungaemia in this group of patients. Similarly, it was concluded that in spite of earlier treatment and regardless of the development of new antifungal agents, the prognosis of invasive fungal infections in patients with haematological malignancies remained poor $[11,12]$. On the other hand, all patients with isolated fungi localised to an i.v. line (whether cannula, CVC or portacath) were successfully managed by administration of an antifungal agent and removal of the line. Fungal pathogens isolated from i.v. lines were different from those causing disseminated fungal infections, being mostly C. parapsilosis and Cr. neoformans from i.v. lines and C. tropicalis and Aspergillus spp. from fungaemias. In agreement with these results, $C$. parapsilosis was reported in severely debilitated subjects in association with contaminated infusions used as parenteral nutrition and in catheter colonisation [15].

Although it is unusual to isolate $C r$. neoformans from intravascular device blood cultures, this finding was recorded in five of the patients in the study. This could be partly explained by the fact that cutaneous cryptococcosis is known to be encountered regularly in immunosuppressed patients [16]. All five cases occurred during a 1-month period and were hospitalacquired, stressing the importance of nosocomial spread of infection. In addition, hospital air has often been found to contain Aspergillus spores, which when inhaled by immunocompromised patients may produce pulmonary infection or disseminated infection, or both. Outbreaks of infection with Aspergillus spp. have occurred in immunocompromised hosts exposed to Aspergillus spores during hospital construction [17]. Continuous reconstruction at our hospital may explain the high frequency of infection with Aspergillus spp. As the majority of fungal infections in the patients $(68.5 \%)$ were hospital-acquired, patients are probably exposed to Aspergillus spp. during their stay in hospital.

As previously reported [2], pneumonia was found to be the commonest fungal infection in these paediatric cancer patients. The isolated pathogen was primarily non-albicans Candida spp. followed by Aspergillus spp. Despite the marked increase in the incidence of infections by Candida spp., candida pneumonia remains a poorly understood entity [18]. No single clinical manifestation is characteristic of this infection. Persistent fever in neutropenic patients is the main sign of disseminated candidosis [18]. 
As it is difficult to interpret the results of the conventional sputum culture because of contamination from the oropharynx, other non-contaminated specimens were obtained in this study. Therefore, fibreoptic bronchoscopy and broncho-alveolar lavage were performed in order to reach a definite microbial aetiology. Fungal infection was diagnosed to be the cause of pneumonia in BAL-positive culture together with the radiological appearance of pulmonary infiltrations. The overall sensitivity of bronchoscopic procedure in the identification of infections in immunocompromised patients is $90 \%$. The exact anatomical origin of specimens, being consistent with the radiological appearance of pulmonary infiltrates, was precisely localised and consequently accurately accessed [19]. A more invasive diagnostic procedure, trans-tracheal aspiration, was applied only if fibreoptic bronchoscopy was not feasible for those immunocompromised patients. Nevertheless, the radiological appearance of pulmonary infiltrates among those immunocompromised patients justified the use of such invasive diagnostic procedures. The isolation of Candida spp. from pulmonary infections was usually secondary to either candida mucositis or oesophagitis.

Thus it is concluded that fungal infections are an important cause of morbidity and mortality in paediatric cancer patients receiving chemotherapy at this institution. Non-albicans Candida spp. are isolated more frequently than $C$. albicans and that probably contributes to the unfavourable outcome observed with disseminated fungal infections in this study. Finally, every effort should be made to prevent fungaemia and fungal pneumonia, as they are associated with a high mortality rate despite early institution of antifungal therapy.

We thank Tarek Mostafa, the paediatric microbiology laboratory technologist, for his enthusiasm and continuous efforts in the field of mycology. Thanks also go to First Monitor, who supplied the kits for Candida species identification.

\section{References}

1. Swerdloff JN, Filler SG, Edwards JE. Severe candidal infections in neutropenic patients. Clin Infect Dis 1993; 17 Suppl 2: S457-S467.

2. Freifeld AG, Walsh TJ, Pizzo PA. Infectious complications in the pediatric cancer patient. In: Pizzo PA, Poplack DG (eds) Principles and practice of pediatric oncology, 3rd edn. Philadelphia, Lippincott-Raven. 1997: 1069-1114.

3. Lanzkowsky P. Supportive care and management of oncologic emergencies. In: Lanzkowsky $P$ (ed) Manual of pediatric hematology and oncology, 3rd edn. San Diego, Academic Press. 2000: 669-717.

4. Wingard JR. Importance of Candida species other than $C$. albicans as pathogens in oncology patients. Clin Infect Dis 1995; 20: $115-125$.

5. Pfaller MA, Jones RN, Messer SA, Edmond MB, Wenzel RP. SCOPE Participant Group. National surveillance of nosocomial bloodstream infection due to species of Candida other than Candida albicans: frequency of occurrence and antifungal susceptibility in the SCOPE Program. Diagn Microbiol Infect Dis 1998; 30: 121-129.

6. Wilson ML, Davis T, Mirrett S et al. Controlled comparison of the BACTEC high-blood-volume fungal medium, BACTEC plus 26 anerobic blood culture bottle, and 10-millilitre isolator blood culture system for detection of fungemia and bacteremia. $J$ Clin Microbiol 1993; 31: 865-871.

7. Waller J, Contant G, Crouzier C, Debruyne M, Koenig H. Evaluation of a new yeast identification system: Fungichrom ${ }^{\mathrm{R}}$ based on chromogenic substrate hydrolysis and carbohydrate assimilation. J Mycol Med 1995; 5: 92-97.

8. Flynn PM, Mariana NM, Rivera GK, Hughes WT. Candida tropicalis infections in children with leukemia. Leuk Lymphoma 1993; 10: 369-376.

9. Girmenia C, Martino P, De Bemrdis F et al. Rising incidence of Candida parapsilosis fungemia in patients with hematologic malignancies: clinical aspects, predisposing factors, and differential pathogenicity of the causative strains. Clin Infect Dis 1996; 23: 506-514.

10. Velasco E, Thuler LC, Martins CA, Nucci M, Dias LM, Goncalves VM. Epidemiology of bloodstream infections at a cancer center. Sao Paulo Med J 2000; 118: 131-138.

11. Viscoli C, Girmenia C, Marinus A et al. Candidemia in cancer patients; a prospective, multicenter surveillance study by the Invasive Fungal Infecton Group (IFIG) of the European Organization for Research and Treatment of Cancer (EORTC). Clin Infect Dis 1999; 28: 1071-1079.

12. Krcmery V, Oravcova E, Spanick S et al. Nosocomial breakthrough fungaemia during antifungal prophylaxis or empirical antifungal therapy in 41 cancer patients receiving antineoplastic chemotherapy: analysis of aetiology, risk factors and outcome. J Antimicrob Chemother 1998; 41: 373-380.

13. Pagano L, Antinori A, Ammassari A et al. Retrospective study of candidemia in patients with hematological malignancies. Clinical features, risk factors and outcome of 76 episodes. Eur J Haematol 1999; 63: 77-85.

14. Krupova Y, Sejnova D, Dzatkova J et al. Prospective study on fungemia in children with cancer: analysis of 35 cases and comparison with 130 fungemias in adults. Support Care Cancer 2000; 8: 427-430.

15. Levin AS, Costa SF, Mussi NS et al. Candida parapsilosis fungemia associated with implantable and semi-implantable central venous catheters and the hands of healthcare workers. Diagn Microbiol Infect Dis 1998; 30: 243-249.

16. Vigouroux S, Morin O, Milpied N, Mahe B, Rapp MJ, Haroussou JL. [Cryptococcus neoformans infection in hematologic malignancies.] Rev Med Interne 2000; 21: 955-960.

17. Andriole T. Infection with Aspergillus species. Clin Infect Dis 1993; 17 Suppl 2: S481-S486.

18. Kiwan E, Anaissie E. Fungal infections complicating neoplastic diseases. In: Sarosi GA, Davies SF (eds) Fungal diseases of the lung, 3rd edn. Philadelphia, Lippincott Williams \& Wilkins. 2000: 219-238.

19. Prakash UBS. Bronchoscopy. In: Albert RK, Spiro SG, Jett JR (eds) Comprehensive respiratory medicine. London, Mosby. 1999: $6.1-6.8$. 